Exercices Exercices de rhétorique

de rhétorique $\quad 8 \mid 2017$

La rhétorique biblique et sémitique

\title{
Comment réaliser des exercices d'analyse rhétorique biblique
}

Roland Meynet

\section{OpenEdition}

1 Journals

Édition électronique

URL : https://journals.openedition.org/rhetorique/503

DOI : $10.4000 /$ rhetorique.503

ISSN : 2270-6909

Éditeur

UGA Éditions/Université Grenoble Alpes

Édition imprimée

ISBN : 978-2-84310-343-8

Référence électronique

Roland Meynet, «Comment réaliser des exercices d'analyse rhétorique biblique », Exercices de rhétorique [En ligne], 8 | 2017, mis en ligne le 25 janvier 2017, consulté le 28 novembre 2021. URL: http://journals.openedition.org/rhetorique/503 ; DOI : https://doi.org/10.4000/rhetorique.503

Ce document a été généré automatiquement le 28 novembre 2021.

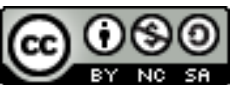

Les contenus de la revue Exercices de rhétorique sont mis à disposition selon les termes de la Licence Creative Commons Attribution - Pas d'Utilisation Commerciale - Partage dans les Mêmes Conditions 4.0 International. 


\title{
Comment réaliser des exercices d'analyse rhétorique biblique
}

\author{
Roland Meynet
}

\section{NOTE DE L'ÉDITEUR}

Pour agrandir les images, cliquer en-dessous sur les mentions « Agrandir » (taille moyenne) ou «Original » (taille réelle).

1 En 2013, nous avons publié un ouvrage intitulé Exercices d'Analyse Rhétorique biblique ${ }^{1}$. Ce n'était pas notre première tentative. En effet, en 1996, j'avais publié un petit volume qui, dans mon intention, ne devait être que le premier d'une série de trois ${ }^{2}$. Une version digitale de ces exercices devint ensuite disponible sur le site de la Société internationale pour l'étude de la Rhétorique Biblique et Sémitique (RBS): «Et maintenant, écrivez pour vous ce cantique." Introduction pratique à l'analyse rhétorique. I. Dictons et proverbes. Cette édition digitale a été retirée en 2013 , après la publication de nos Exercices d'Analyse Rhétorique, car ils étaient devenus dépassés.

Or nous n'avons pas été les premiers - et de loin - à proposer des exercices à ceux qui veulent apprendre à analyser les textes bibliques selon les lois de la rhétorique biblique. Il y a bientôt deux siècles, Thomas Boys - qui avec John Jebb mérite le nom de fondateur de l'analyse rhétorique biblique, ou de découvreur de la rhétorique biblique ${ }^{3}$ - achevait son second livre ${ }^{4}$ avec un cinquième et dernier appendice intitulé « Exercises for students ». Il valait la peine de traduire en français les quatre pages de cet appendice, qui fourniront le point de départ de notre réflexion. 


\section{Les exercices de Thomas Boys}

\section{Énoncé de l'exercice : Thomas Boys, $A$ Key to the Book of the Psalms (18255)}

«Certains biblistes pourraient désirer étudier par eux-mêmes les parallélismes du Texte Sacré. Dans ce cas, ils seront susceptibles, d'une part, de se fourvoyer dans des découvertes imaginaires; et, d'autre part, de se décourager à cause d'essais infructueux, et donc d'abandonner la recherche.

Il peut donc être utile de signaler quelques passages qui ont déjà été analysés, de sorte que l'étudiant puisse commencer par ces textes pour s'exercer. Je les donne sans solution, alternant ceux qui sont faciles et ceux qui sont plus difficiles, en recommandant de prêter une attention particulière au texte original, dans les cas où l'arrangement n'est pas immédiatement perceptible [...].

1. Arrangez, en un parallélisme inversé [= construction concentrique] de huit membres [= unités], le passage qui commence par «Et il vint dans une maison » (Mc 3,19-4,16).

2. Arrangez le passage qui commence par « Écoutez-moi » (Mc 7,14-16).

[p. 237]

3. Découvrez l'arrangement des termes suivants: "Quelles pierres et quelles constructions ; Il ne restera pas pierre sur pierre, qui ne soit détruite » $(\mathrm{Mc} 13,1.2)$.

4. « Race de vipères, etc. ...... enfants à Abraham » (Mt 3,7-9).

5. «On lui amena, etc. ...... le muet parla » (Mt 9,32-33).

6. Jn 4,50 ; et 9,4 .

7. « Mon temps ", etc. (Jn 7,6-8).

8. «C'est toi qui es son disciple », etc. Jn $9,28,29$

9. «Vous savez», etc. Ac 20,18-35, une composition accomplie. Essayez d'abord 26-31; ou 22-32.

10. $\mathrm{Rm} 2,6-11$.

11. $\mathrm{Rm} 2,25$.

12. $\mathrm{Rm} \mathrm{5,12-21.} \mathrm{Ce} \mathrm{passage} \mathrm{doit} \mathrm{être} \mathrm{arrangé} \mathrm{selon} \mathrm{la} \mathrm{forme} \mathrm{suivante.} \mathrm{Alors} \mathrm{vous} \mathrm{le}$ comprendrez pour la première fois.

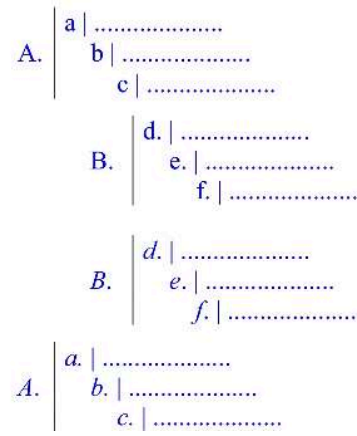

13. $\mathrm{Rm} 6,8-11$.

14. $\mathrm{Rm} \mathrm{9,21-24.}$

[p. 238]

15. Rm 13,1-6.

16. « Si un frère, etc. ...... sanctifiée par le mari » 1Co 7,12-14.

17. 1Co 7,34.

18. 1Th 5, 5. Rm 13,12.

19. "Voici que des jours viennent", etc. He 8,8-12. Ce passage comprend deux arrangements, dont la caractéristique est remarquablement semblable [remarkably resembling each other in character]. 
20. Ap 2,2.3.

21. 1P 3,8-12; et "Revêtez-vous tous d'humilité », etc. 1P 5,5-7. La caractéristique

[The character] de ces deux passages est la même.

22. Indiquez quel est le point commun entre trois phrases dans 1P 1,3-5.

23. Même chose en 1P 2,18-21.

24. Is 13,15 .

25. Is $24,1-5$ [Boys écrit seulement « xiii. $-5 »]$.

26. Is 41, 1-10 [Boys écrit seulement « xli. $-10 »]$.

27. Ps 49,4 (selon la numérotation de la Bible hébraïque).

28. Ps 86,5 .

29. «Mon cœur exulte », etc. $1 \mathrm{~S} 2,1$.

30.

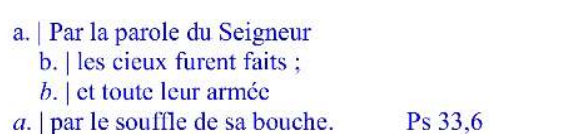

Les choses faites, en b. et $b$. ; et les instruments ou Agents (la Parole et l'Esprit de Dieu) en a. et $a$. [p. 239]

a. Fais-moi vivre, Seigneur

b. | à cause de ton nom ;

b. $\mid$ à cause de ta justice

a. | tire mon âme de l'angoisse. Ps 143,11

En a. et $a$. la demande [the petition]; en b. et $b$. la raison ou le motif [the plea or inducement].

D'où apparaît-il que ces deux dispositions sont erronées et irrecevables?

31. Griesbach intervertit Mt 23,13 et 14. Montrez que le parallélisme confirme cette correction.

5 S'il le désire, l'étudiant peut s'exercer avec ces quelques exemples. En conclusion je me propose d'offrir, à titre d'encouragement, une Bible hébraïque et un Testament grec, qui seront attribués respectivement aux deux meilleurs concurrents. On peut m'envoyer les exercices, rédigés entièrement, à tout moment avant le mois d'août 1826, en spécifiant le nom et l'adresse de l'auteur; si on préfère ne pas fournir ces coordonnées, on pourra utiliser un verset de l'Écriture comme signe de reconnaissance. Si nécessaire, une annonce sera insérée dans le Missionary Register, à paraître fin septembre 1826, où seront publiés les noms des candidats retenus. Le concours est ouvert aussi bien aux étudiants étrangers qu'aux Britanniques; et les communications peuvent être rédigées en latin, en français ou en anglais. 34, Bouverie Street, Fleet Street. »

\section{Solution}

$6 \quad$ Nous ne savons pas si le concours lancé par Boys a eu beaucoup de succès. En tout cas, je n'ai pas trouvé trace de quelque annonce que ce soit dans la revue Missionary Register; et les prix pour les deux meilleurs concurrents ne semblent pas avoir été jamais attribués.

7 Notre "Société internationale pour l'étude de la Rhétorique Biblique et Sémitique " (RBS) avait pensé reprendre le flambeau, en relançant le concours de Boys, avec les prix qu'il y avait attachés.

8 Toutefois, ayant entrepris de faire ses exercices, j'ai pu constater que, si certains textes avaient été bien délimités, d'autres au contraire ne l'étaient pas du tout. Comme Boys s'était dispensé de fournir un corrigé, nous ne pouvons pas savoir les raisons de ses découpages. On risquerait donc de fourvoyer les étudiants en leur conseillant de faire ces exercices. À titre d'exemple, nous examinerons les trois premiers.

9 Voyons d'abord le deuxième et le troisième exercice. 
Énoncé de Thomas Boys : 2. Arrangez le passage qui commence par "Écoutez-moi » (Mc 7,14-16).

La réécriture du texte suffira à montrer que Boys en a bien identifié les limites ${ }^{7}$ :

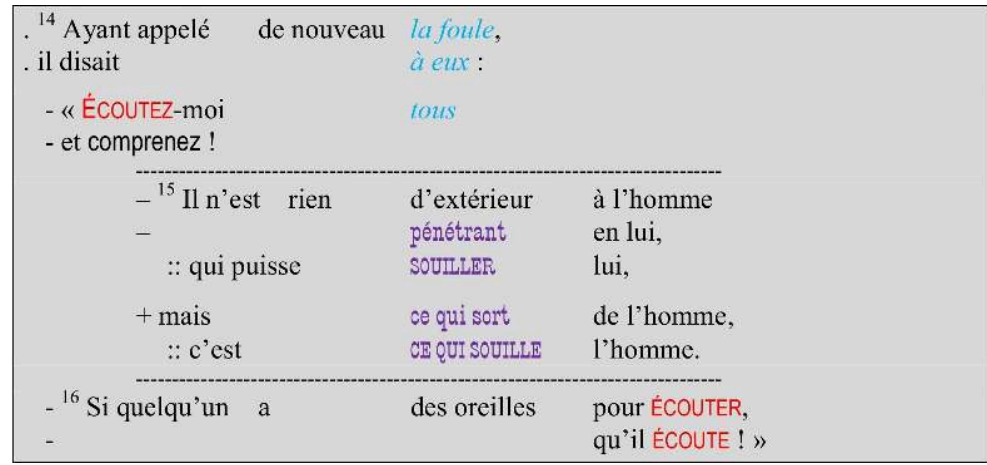

Énoncé de Thomas Boys : 3. Découvrez l'arrangement des termes suivants : "Quelles pierres et quelles constructions; il ne restera pas pierre sur pierre, qui ne soit détruite " (Mc 13,1.2).

De nouveau, la réécriture du texte suffit à montrer que Boys en a bien identifié les limites $^{8}$ :

\begin{tabular}{|c|c|c|c|}
\hline $\begin{array}{l}+{ }^{1} \text { Et allant } \\
=d i t\end{array}$ & $\begin{array}{l}\text { lui } \\
\text { à lui }\end{array}$ & $\begin{array}{l}\text { hors du temple } \\
\text { un }\end{array}$ & de ses disciples : \\
\hline $\begin{array}{l}\text { - « Maître, } \\
\text { : et }\end{array}$ & REGARDE & $\begin{array}{l}\text { quelles } \\
\text { quelles }\end{array}$ & $\begin{array}{l}\text { pierres } \\
\text { constructions! }\end{array}$ \\
\hline $\begin{array}{l}={ }^{2} \text { Et Jésus } \\
\quad: \text { : } \\
\quad \text { - Ne sera pas laissée } \\
\quad \text { qui ne soit détruite. }\end{array}$ & $\begin{array}{l}\text { dit } \\
\text { «VOIS-TU } \\
\text { ici }\end{array}$ & $\begin{array}{l}\text { à lui : } \\
\text { ces grandes } \\
\text { une pierre }\end{array}$ & $\begin{array}{l}\text { constructions? } \\
\text { sur une pierre }\end{array}$ \\
\hline
\end{tabular}

Revenons maintenant au premier exercice :

Énoncé de Thomas Boys : 1. Arrangez, en un parallélisme inversé [introverted parallelism, construction concentrique, ou spéculaire] de huit membres [members, unités], le passage qui commence par «Et il vint dans une maison » $(\mathrm{Mc}$ 3,20-4,1).

Selon mon analyse, ces versets correspondent aux trois derniers passages d'une séquence qui en comporte cinq ${ }^{9}$; en outre, le premier verset du chapitre 4 n'appartient pas à cette séquence mais à la suivante ${ }^{10}$.

Il n'est pas possible de savoir quels étaient les huit membres que Boys distinguait dans ces dix-sept versets.

En somme, il en va des exercices proposés par Boys comme des textes qu'il étudie tout au long de son livre: si certaines de ses analyses sont tout à fait convaincantes, d'autres le sont beaucoup moins ou sont franchement erronées, ce qui ne saurait étonner chez un pionnier comme lui.

Passons maintenant à la deuxième partie de cet article, la préparation d'un exercice d'analyse rhétorique. Le choix s'est porté sur le Magnificat (Lc 1,46b-55), pour trois raisons principales: d'abord parce que c'est un texte court, puis parce que c'est un texte poétique, donc plus facile à analyser, enfin parce qu'il contient des segments variés. 


\section{Préparer un exercice sur le magnificat}

La première chose à faire est de fournir le texte à l'apprenti : le texte original - grec en l'occurrence - et aussi une traduction littérale, de sorte qu'il puisse travailler directement sur le texte, sans devoir le taper. Pour le Magnificat, on a ajouté quelques traductions parmi les plus utilisées, pour que l'apprenant puisse constater la distance entre l'original et ces traductions, en particulier la traduction liturgique qui « défigure » le texte, c'est-à-dire qui en fait disparaître la «figure » ou la composition originale.

\section{Texte et traductions}

\section{Texte grec}

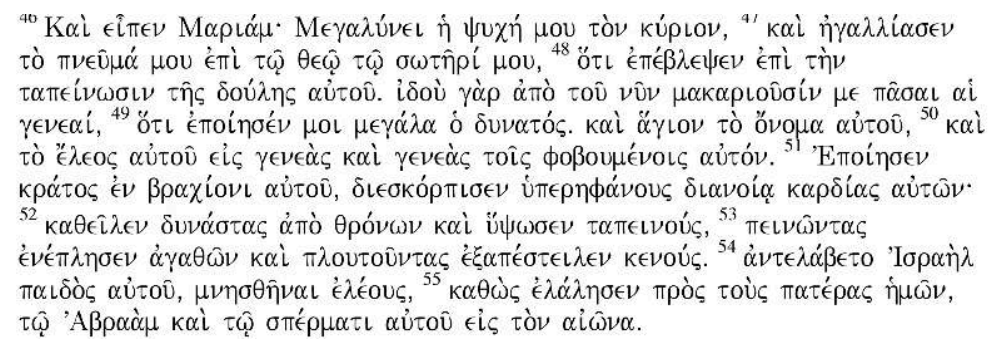

\section{Traduction liturgique}

Voici la traduction utilisée dans la liturgie catholique à l'heure actuelle.

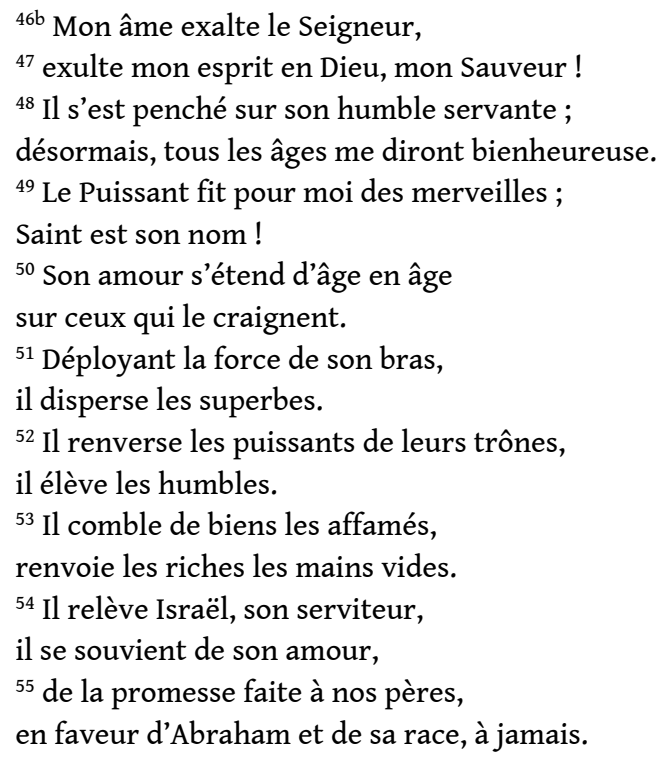

Traduction de la Bible de Jérusalem (1998)

19 C'est la traduction française la plus utilisée (en notes les différences avec la première édition en un seul volume de 1956).

Mon âme exalte le Seigneur,

${ }^{47}$ et mon esprit tressaille de joie en Dieu

mon Sauveur,

${ }^{48}$ parce qu'il a jeté les yeux sur l'abaisse- 
ment de sa servante ${ }^{11}$.

Oui, désormais toutes les générations

me diront bienheureuse,

${ }^{49}$ car le Tout-Puissant a fait pour moi de

grandes choses.

Saint est son nom,

${ }^{50}$ et sa miséricorde s'étend d'âge en âge

sur ceux qui le craignent.

${ }^{51}$ Il a déployé la force de son bras,

il a dispersé les hommes au cœur

superbe.

${ }^{52} \mathrm{Il}$ a renversé les potentats de leurs trô-

nes et élevé les humbles,

${ }^{53} \mathrm{Il}$ a comblé12 de biens les affamés et ren-

voyé les riches les mains vides.

${ }^{54}$ Il est venu en aide ${ }^{13}$ à Israël son serviteur,

se souvenant de sa miséricorde,

55 - selon qu'il l'avait annoncé ${ }^{14}$ à nos

pères -

en faveur d'Abraham et de sa postérité $e^{15}$

à jamais !

La disposition typographique a été scrupuleusement respectée (mais les italiques qui signalent les références aux textes de l'Ancien Testament ont été supprimées).

\section{Traduction très littérale}

${ }^{46 b}$ Grandit mon âme le Seigneur ${ }^{47}$ et jubile mon esprit en Dieu mon sauveur ${ }^{48}$ parce qu'il a regardé l'humiliation de sa servante voici en effet à partir de maintenant me diront-heureuse tous les âges ${ }^{49}$ parce qu'il a fait à moi de grandes (choses) le Puissant et saint son nom ${ }^{50}$ et sa miséricorde d'âge en âge sur les craignant lui ${ }^{51}$ il a fait force par son bras il a dispersé les orgueilleux en pensée de leur cœur ${ }^{52}$ il a renversé les puissants des trônes et il a élevé les humiliés ${ }^{53}$ les affamés il a remplis de biens et les riches il a renvoyé vides ${ }^{54}$ il a secouru Israël son serviteur pour se rappeler la miséricorde ${ }^{55}$ comme il avait dit à nos pères en-faveur-d'Abraham et de sa descendance à jamais.

\section{B. Au travail !}

Si vous ne connaissez pas, ou pas suffisamment le grec, travaillez sur la traduction littérale.

Commencer par l'analyse syntaxique permet déjà un découpage en propositions qui forment des phrases. Dans le cas du Magnificat, ce sera important pour pouvoir critiquer en connaissance de cause les traductions cités plus haut, en particulier la traduction liturgique.

\section{Analyse syntaxique}

\section{a. Consigne}

Les anciens manuscrits, onciaux ou minuscules, ne comportaient pas de ponctuation. Le texte grec ci-dessus est celui de la $28^{\mathrm{e}}$ édition du Novum Testamentum Graece, de NestleAland, qui a ponctué le texte. Vous devrez donc faire pour la traduction littérale le travail d'un éditeur moderne : ajouter les signes de ponctuation. Cela suppose que vous 
ayez fait l'analyse syntaxique du texte, c'est-à-dire que vous le divisiez en phrases, et les phrases en propositions, principale et subordonnée(s).

\section{b. Solution} servante », car ce qui suit est clairement une autre phrase. En revanche, où finit la deuxième phrase ? En effet, « et saint son nom » qui commence par un coordonnant pourrait être une phrase coordonnées à la précédente; et de même pour la phrase nominale suivante (50), même s'il pourrait sembler curieux que deux phrases nominales soient coordonnées à une phrase verbale dont le verbe est à l'aoriste (traduit par un passé). commençant pas par un coordonnant, chacun forme une seule phrase. Les deux derniers versets forment une seule phrase complexe qui ne commence pas non plus par un coordonnant. 


\section{Segmentation (1). Proposition par proposition}

31 Sans le dire, on commencera l'analyse rhétorique "par le bas ", c'est-à-dire à partir de l'unité de base qu'est le "membre" et la première unité proprement rhétorique, c'est-à-dire compositionnelle qu'est le «segment 》 (voir Traité, 126).

La division syntaxique est primordiale, mais elle ne coïncide pas toujours avec la division rhétorique. En effet, si, le plus souvent, le «membre » est une proposition, il arrive aussi qu'un membre soit seulement un syntagme, c'est-à-dire une partie d'une proposition.

On appelle "segmentation» la division en «segments». Or le segment est une unité textuelle qui compte un, deux ou trois «membres» (sur la définition du membre, étudiez, si ce n'est déjà fait Traité, «Le membre », 136-147). C'est pourquoi la première opération de l'analyse rhétorique est de diviser le texte en « membres ».

Après quoi, on cherche à regrouper les membres en segments.

\section{a. Consigne}

Réécrivez d'abord le texte, une proposition par ligne, en séparant les phrases par une ligne blanche.

Vous pouvez aussi proposer une première ponctuation, mettant un point entre chaque phrase et séparant les phrases dont vous pouvez être sûr par une ligne blanche.

\section{b. Solution}

Le texte comprend 16 propositions, que l'on peut regrouper, provisoirement, en 7 phrases :

${ }^{46 b}$ Grandit mon âme le Seigneur

${ }^{47}$ et jubile mon esprit en Dieu mon sauveur,

${ }^{48}$ parce $\mathbf{q u}$ 'il a regardé l'humiliation de sa servante.

Voici en effet à partir de maintenant me diront-heureuse tous les âges,

${ }^{49}$ parce $\boldsymbol{q u}$ 'il a fait à moi de grandes (choses) le Puissant

et saint son nom

${ }^{50}$ et sa miséricorde d'âge en âge sur les craignant lui.

${ }^{51} \mathrm{Il}$ a fait force par son bras.

il a dispersé les orgueilleux en pensée de leur cœur.

${ }^{52} \mathrm{Il}$ a renversé les puissants des trônes

et il a élevé les humiliés.

${ }^{53}$ Les affamés il a remplis de biens

et les riches il a renvoyé vides.

${ }^{54} \mathrm{Il}$ a secouru Israël son serviteur,

pour se rappeler la miséricorde,

${ }^{55}$ comme il l'avait dit à nos pères en-faveur-d'Abraham et de sa descendance à jamais.

On met les propositions qui commencent par une conjonction de subordination ou par le coordonnant « et » avec la proposition qui la précède.

\section{Segmentation (2). Regroupez les membres en segments}

Il n'est pas dit qu'il faille commencer par le début du texte. Allez d'abord au plus évident et au plus sûr. 


\section{a. Consigne. Les deux segments les plus évidents}

\section{b. Solution}

44 Passons alors au verset 52 : premier membre. segments.

Quels sont les deux segments bimembres dont on peut penser qu'ils sont indubitables? Réécrivez-les en alignant verticalement leurs termes, et en visualisant par quelque artifice typographique, les correspondances entre les deux membres. N'oubliez pas de justifier votre décision par écrit.

Les segments bimembres indubitables sont ceux des versets 52 et 53 :

\begin{tabular}{|c|c|c|c|}
\hline & $\pi \epsilon \iota \nu(\hat{\omega} \nu \tau \alpha \varsigma$ & ENEПAHЕEN & $\dot{\alpha} \gamma \alpha \theta \omega \bar{\omega} \nu$ \\
\hline$+\kappa \alpha \grave{\imath}$ & 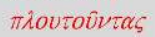 & EEAПEZTEI $\Lambda E N$ & кєvoúç. \\
\hline$+{ }^{53}$ & Les affamés & IL A REMPLIS & de biens \\
\hline+ et & les riches & IL A RENVOYÉS & vides. \\
\hline
\end{tabular}

Les deux membres sont coordonnés. L'opposition entre les compléments d'objet direct, « les affamés » et « les riches », est « oblique »; l'opposition directe serait « affamés »«rassasiés» ou «riches» - "pauvres». De même le terme directement opposé au verbe «remplir» est «vider», mais l'image se retrouve dans le dernier terme. L'opposition des deux membres est indéniable.

43 D'avoir identifié ce segment fournit déjà deux limites (même si c'est encore provisoire) : la fin du segment précédent et le début du suivant.

\begin{tabular}{|c|c|c|}
\hline 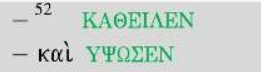 & 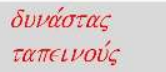 & 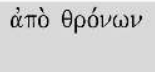 \\
\hline $\begin{array}{l}\text { - }{ }^{52} \text { IL A RENVERSÉ } \\
\text { - et IL A ÉLEVÉ }\end{array}$ & $\begin{array}{l}\text { les puissants } \\
\text { les humiliés. }\end{array}$ & des trônes \\
\hline
\end{tabular}

De nouveau les deux membres sont coordonnés. De nouveau l'opposition entre les termes est oblique : l'antonyme de "élever » est "abaisser ", mais « renverser » est appelé par l'image du roi mis à bas de son trône. On peut comprendre que les personnages dont parle le second membre sont «humiliés » par «les puissants » du

S'il s'agit bien de deux segments bimembres, nous savons que la fin de 51 marque la fin d'un segment et que le début de 54 marque le début d'un autre segment.

\section{c. Consigne. En reprenant par la fin}

Vous avez certainement identifié facilement les deux segments bimembres les plus évidents. Et cela vous donnait - au moins provisoirement - les limites d'autres

Il est en outre deux autres limites de segments, celles-ci indéniables : le début du texte et sa fin! Maintenant, vous pourriez donc commencer par la fin. N'oubliez pas que l'analyse syntaxique que vous avez faite devrait vous aider à diviser en membres, à partir du dernier mot du cantique.

4 N'oubliez pas ce que vous avez lu dans le Traité sur la définition du membre (spécialement, 144-147). Lisez aussi les pages sur la définition du «terme» (148-150). 
Pour la dernière phrase du chant vous est fournie une traduction encore plus littérale que celle qui a été donnée au début de l'exercice :

${ }^{54} \mathrm{Il}$ a secouru Israël serviteur de lui pour se rappeler la miséricorde ${ }^{53}$ comme il avait dit aux pères de nous en-faveur-d'Abraham et de la descendance de lui à jamais.

\section{d. Solution}

\begin{tabular}{|c|c|c|c|}
\hline $\begin{array}{l}+{ }^{54} \dot{\alpha} \nu \tau \tau \epsilon \lambda \dot{\alpha} \beta \in \tau o \\
+\mu \nu \eta \theta \dot{\eta} \nu \alpha \iota\end{array}$ & 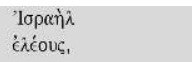 & $\pi \alpha L \delta \delta$ ç & aútoù \\
\hline 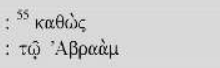 & 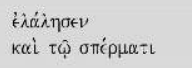 & 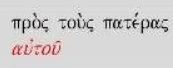 & 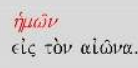 \\
\hline $\begin{array}{l}+{ }^{54} \mathrm{Il} \text { a secouru } \\
+ \text { pour se rappeler }\end{array}$ & $\begin{array}{l}\text { Israël } \\
\text { la miséricorde, }\end{array}$ & serviteur & delui \\
\hline $\begin{array}{l}:{ }^{55} \text { comme } \\
: \text { en-faveur-d'Abraham }\end{array}$ & $\begin{array}{l}\text { il avait dit } \\
\text { et de la descendance }\end{array}$ & $\begin{array}{l}\text { aux pères } \\
\text { de lui }\end{array}$ & $\begin{array}{l}\text { de nous } \\
\text { à jamais. }\end{array}$ \\
\hline
\end{tabular}

Cette traduction, qui calque le grec, considère les pronoms au génitif (compléments de nom) comme autant de termes (en italiques rouges dans la réécriture ci-dessus). En hébreu au contraire, ces pronoms sont très courts et affixes, faisant partie du terme auquel ils sont accolés.

51 La question de savoir si ces pronoms doivent être considérés comme des termes est délicate. Les pronoms de première personne sont courts (monosyllabiques) et posent moins de problème; en revanche ceux de deuxième et de troisième personne étant dissyllabiques, devraient être comptés comme un terme du point de vue du rythme.

Dans la traduction littérale donnée au début, ces pronoms ont été traduits par des possessifs et on considérera donc le nom précédé de son pronom possessif comme un seul terme :

$\begin{array}{lll}+{ }^{54} \text { Il a secouru } & \text { Israël } & \text { son serviteur, } \\ + \text { pour se rappeler } & \text { la miséricorde, } & \\ :{ }^{55} \text { comme } & \text { il avait dit } & \text { à nos pères } \\ : \text { en-faveur-d'Abraham } & \text { et de sa descendance } & \text { à jamais. }\end{array}$

Les deux premières propositions sont la principale et la finale (54a et 54b) dont le rythme est de $3+2$ termes. La comparative qui suit compte six termes et par conséquent elle est divisée en deux membres comptant chacun trois termes.

Les correspondances entre les membres du même segment ne sont pas évidentes comme elles l'étaient pour les deux bimembres que vous avez analysés précédemment. On pourra dire que pour le premier segment, «Israël» et «miséricorde » sont complémentaires :

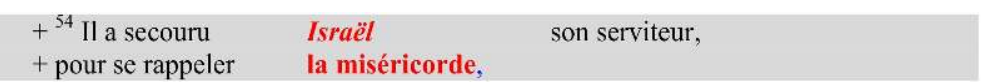

«Israël » est le bénéficiaire de la «miséricorde »; en d'autres termes, «Israël » est l'objet tandis que « la miséricorde » est le sujet. Il pourrait sembler curieux que le texte ne dise pas «sa miséricorde» (comme font toutes les traductions), comme si «la miséricorde " était en quelque sorte une personnification divine, tout au moins un attribut divin.

Quant au second segment :

$\begin{array}{lll}:{ }^{55} \text { comme } & \text { il avait dit } & \text { à nos pères } \\ : \text { en-faveur-d'Abraham } & \text { et de sa descendance } & \text { à jamais. }\end{array}$

on remarquera seulement une correspondance entre «nos pères » et «Abraham et sa descendance » qui indiquent les mêmes personnes. 
Quant à l'ensemble de ces deux segments - dont on voit bien qu'ils vont ensemble, puisqu'ils ne forment qu'une seule phrase - on pourra penser qu'ils appartiennent à un même « morceau ».

$\begin{array}{lll}+{ }^{54} \text { II A SECOIRI } & \text { ISRAËL } & \text { son serviteur, } \\ + \text { pour SE RIPPELLII } & \text { la miséricorde, } & \\ :{ }^{55} \text { comme } & \text { II. ANIT DIT } & \text { à nos pères } \\ : \text { en-faveur-d'ABRAHAM } & \text { ET DE SA DESCENDANCE à jamais. }\end{array}$

Les trois verbes ont le même sujet (Dieu). Aux extrémités «Israël» est «la descendance » d'« Abraham ». Israël est qualifié d'abord par sa relation avec Dieu dont il est le « serviteur » (54a) puis par sa relation avec celle qui parle (et ceux qui parlent quand ils reprennent son chant), dont ils sont «les pères». Par ailleurs, il faut noter que le terme grec traduit par « serviteur ", pais, signifie aussi « enfant »; pour le rendre plus exactement, en respectant l'ambiguïté de l'original, il faudrait dire "son garçon ». Ainsi apparait mieux le rapport entre les termes finaux des premiers membres de chaque segment : Israël est fils de Dieu, et père de ceux qui appartiennent à son peuple, Marie qui prononce ces paroles, et tous ceux qui les reprennent jusqu'à nos jours, qui se reconnaissent ainsi fils d'Abraham, faisant partie du peuple d'Israël.

Qui veut préparer un exercice, doit en faire à mesure un "corrigé" (ou une "solution", si on préfère parler d'énigme) très précis. Pour donner un exemple de cette nécessité absolue, c'est en rédigeant la solution de l'énigme que j'avais proposée en conseillant de partir de la fin du texte, que j'ai réalisé qu'il me fallait affronter la question délicate des pronoms au génitif. Il m'a donc fallu reformuler les consignes de manière plus précise.

60 J'ai aussi été amené à corriger la traduction littérale donnée en commençant. En effet, il fallait éviter d'utiliser la même préposition "pour» au début de la proposition "pour se rappeler la miséricorde " et au début du dernier membre "pour Abraham "; c'est pourquoi j'ai traduit le datif de "Abraham " par "en-faveur-d" (avec les tirets pour qu'on n'aille pas imaginer que c'est un terme!). C'est aussi pourquoi j'ai corrigé « sa miséricorde " par « la miséricorde », car ce terme n'est pas suivi du pronom autou! Du reste, le terme n'ayant pas d'article, on aurait pu aller jusqu'à le traduire «se souvenir de miséricorde »...

\section{e. Consigne. Et en repartant du début}

Et maintenant que vous avez déjà identifié quatre segments, vous pouvez repartir du début, puisque le début du chant marque celui d'un premier segment. Comme pour la fin du chant, n'oubliez par l'analyse syntaxique. Quelle est la première phrase du texte et combien de propositions contient-elle? Faites la réécriture du texte, accompagnée de la justification écrite de votre analyse rhétorique.

\section{f. Solution}

\begin{tabular}{|c|c|c|c|}
\hline 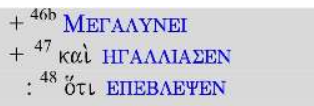 & 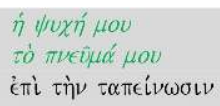 & 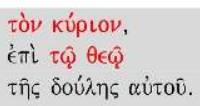 & 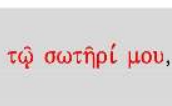 \\
\hline 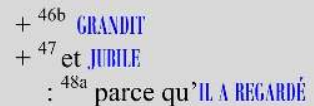 & $\begin{array}{l}\text { mon âme } \\
\text { mon esprit } \\
\text { l'humiliation }\end{array}$ & $\begin{array}{l}\text { le Seigneur } \\
\text { en Dieu } \\
\text { de sa servante. }\end{array}$ & mon sauveur, \\
\hline
\end{tabular}

62 Trois propositions forment une seule phrase: deux principales coordonnées qui régissent une causale. Il s'agit d'un trimembre du type AA'B, car les deux premiers membres sont parallèles tandis que le troisième membre est différent: les deux 
premiers expriment l'action présente de la locutrice envers Dieu, et le troisième en donne la raison qui est l'action passée de Dieu pour elle.

On a donc une nouvelle limite, le début de 49.

\section{g. Consigne. Et le reste?}

Voyons les portions de texte qui n'ont pas encore été analysées :

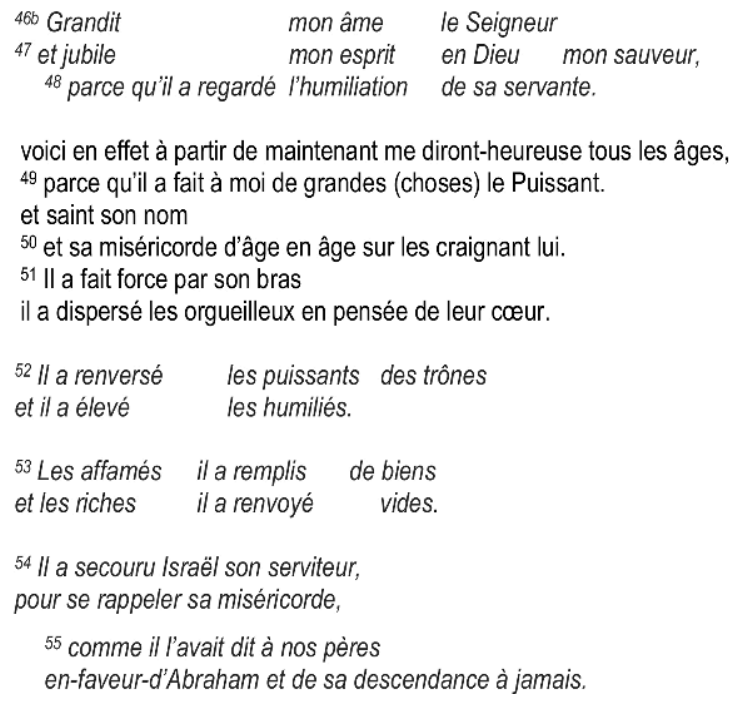
c'est là une caractéristique des textes sémitiques. Un exemple, choisi au hasard! La séquence $\mathrm{C} 1$ de l'évangile de Marc $(10,1-52)$ comprend 12 " passages »; 9 commencent par kai (" et»), deux par de (un autre "coordonnant» qui se met toujours après le premier mot) et un seul ne commence pas avec un coordonnant ${ }^{16}$. Ainsi la controverse sur le divorce, l'accueil des enfants, l'appel du riche, la demande des fils de Zébédée, entre autres, qui sont autant de récits isolables, commencent avec kai (« et »); tous ces coordonnants sont supprimés dans les traductions de la Bible de Jérusalem et de la Traduction Ecuménique de la Bible ; Osty au contraire, qui est très littéral, les maintient.

\section{h. Solution}

Essayons donc ce qui vient après le premier trimembre (46b-48a) :

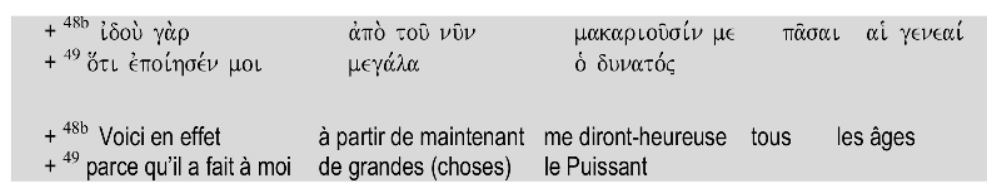


Ce sont deux propositions, la principale suivie d'une causale. La principale est bien longue : elle comprend cinq termes (ou quatre si on ne considère pas "tous » comme un terme). On peut donc essayer de la couper en deux :

$\begin{array}{cll}+{ }^{48 b} \text { Voici en effet } & \text { à partir de maintenant } & \\ + \text { me diront-heureuse } & \text { tous les âges } & \\ :{ }^{49} \text { parce qu'il a fait à moi } & \text { de grandes (choses) } & \text { le Puissant. }\end{array}$

Si l'on fait ainsi, la fin des deux premiers membres se correspond. Ils indiquent le temps : point de départ avec «à partir de maintenant » et point d'arrivée, pour ainsi dire, qui s'étend à l'infini.

Le début du segment ne pose pas de problème, si nous tenons que le premier trimembre (46b-48a) a bien été identifié. Pour la fin au contraire, on peut se demander si la suite, « et saint son nom... », ne pourrait faire partie de la même unité, puisqu'elle commence par « et ». Affaire à suivre!

Et maintenant reprenons par la fin :

\begin{tabular}{|c|c|c|c|c|}
\hline $\begin{array}{l}+51 \\
+\end{array}$ & 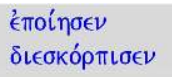 & 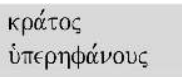 & 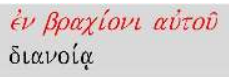 & $\kappa \alpha \rho \delta i \alpha \varsigma \alpha \dot{\tau} \tau \hat{\omega} \nu$ \\
\hline $\begin{array}{l}+51 \\
+\end{array}$ & $\begin{array}{l}\text { IL A FAIT } \\
\text { IL A DISPERSÉ }\end{array}$ & $\begin{array}{l}\text { force } \\
\text { les orgueilleux }\end{array}$ & $\begin{array}{l}\text { par son bras } \\
\text { en pensée }\end{array}$ & de leur cour. \\
\hline
\end{tabular}

Du point de vue syntaxique, ces deux membres ne sont pas coordonnés, mais du point de vue rhétorique ils sont parallèles. Ils commencent en effet par un verbe à l'aoriste (traduit par un passé) et ils s'achèvent par des compléments semblables qui opposent des parties du corps : le «bras » de Dieu, c'est-à-dire son action visible, et le « cœur » des orgueilleux qui est le siège de leurs pensées, de leurs intentions cachées. Il est donc raisonnable de tenir ces deux membres pour un seul segment.

\section{i. Consigne. Et maintenant, le reste du reste!}

Que reste-t-il ? Quelle est la caractéristique de ce dernier reste ? Comment l'analyser? N’oubliez pas ce que vous avez lu dans le Traité sur le rythme (p. 144-147).

\section{j. Solution}

\begin{tabular}{|c|c|c|c|}
\hline 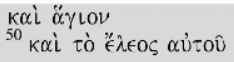 & 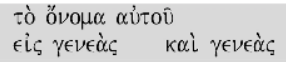 & 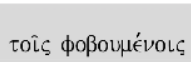 & $\alpha$ นั่óv \\
\hline${ }^{49 b}$ et saint & son nom & & \\
\hline${ }^{50}$ et sa miséricorde & d'âges & sur les craignant & lui. \\
\hline
\end{tabular}

5 Il s'agit de deux propositions nominales (les seules de tout le texte). La première proposition comprend deux termes; avec quatre termes la seconde est deux fois plus longue. Par ailleurs, "les craignant » est un participe suivi d'un complément d'objet direct; il a valeur verbale plus que nominale et le complément pourrait donc être considéré comme une proposition.

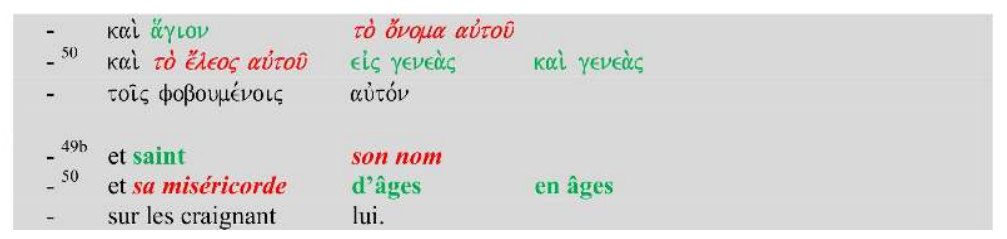

Il semble donc que l'on ait affaire à un segment trimembre. Les deux premiers membres, qui commencent tous deux par le coordonnant «et", ont la même construction syntaxique, bien que leurs termes soient inversés : «son nom » et «sa 
miséricorde » sont sujets, "saint » et « d'âge en âge " prédicats. Alors que l'acteur des deux premiers membres est le Seigneur, celui du troisième membre est ceux qui le craignent. Ce dernier membre exprime l'attitude de respect de ceux qui ont bénéficié de la sainteté et de la miséricorde de «lui », c'est-à-dire du "Saint» et du Miséricordieux. Sous cet aspect, le trimembre est du type AA'B et il faut le réécrire ainsi :

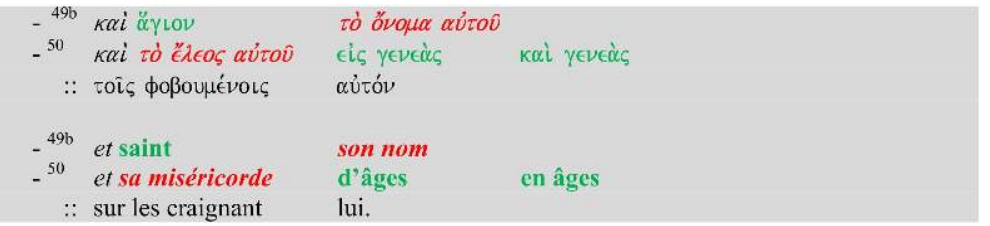

\section{Regrouper les segments en morceaux et en parties}

\section{a. Consigne}

Recopiez les réécritures des segments identifiés jusqu'à présent.

- Séparez-les par une ligne blanche.

- Sélectionnez tout le texte, puis mettez-le en noir, en romain (sans gras, italiques, capitales et petites capitales) et dans le même caractère (Times).

- Alignez les termes verticalement, autant que possible ; pour pouvoir le faire plus facilement, mettez le texte en corps 12 .

\section{b. Solution}

\begin{tabular}{|c|c|c|c|}
\hline $\begin{array}{l}+{ }^{46 \mathrm{~b}} \text { Grandit } \\
+{ }^{47} \text { et jubile } \\
\quad:{ }^{48 \mathrm{a}} \text { parce qu'il a regardé }\end{array}$ & $\begin{array}{l}\text { mon âme } \\
\text { mon esprit } \\
\text { l'humiliation }\end{array}$ & $\begin{array}{l}\text { le Seigneur } \\
\text { en Dieu } \\
\text { de sa servante. }\end{array}$ & mon sauveur, \\
\hline $\begin{array}{l}+{ }^{48 b} \text { Voici en effet } \\
+ \text { me diront-heureuse } \\
\quad:{ }^{49} \text { parce qu'il a fait à moi }\end{array}$ & $\begin{array}{l}\text { à partir de maintenant } \\
\text { tous } \\
\text { de grandes (choses) }\end{array}$ & $\begin{array}{l}\text { les âges } \\
\text { le Puissant. }\end{array}$ & \\
\hline $\begin{aligned}-{ }^{49 b} & \text { Et saint } \\
-{ }^{50} & \text { et sa miséricorde } \\
:: & \text { sur les craignant }\end{aligned}$ & $\begin{array}{l}\text { son nom } \\
\text { d'âge } \\
\text { lui. }\end{array}$ & en âge & \\
\hline $\begin{array}{ll}+{ }^{51} & \text { Il a fait } \\
+ & \text { il a dispersé }\end{array}$ & $\begin{array}{l}\text { force } \\
\text { les orgueilleux }\end{array}$ & $\begin{array}{l}\text { par son bras } \\
\text { en pensée }\end{array}$ & de leur cœur. \\
\hline $\begin{array}{l}{ }^{52} \text { Il a renversé } \\
\text { - et il a élevé }\end{array}$ & $\begin{array}{l}\text { les puissants } \\
\text { les humiliés. }\end{array}$ & des trônes & \\
\hline $\begin{array}{ll}+{ }^{53} & \text { Les affamés } \\
+ \text { et } & \text { les riches }\end{array}$ & $\begin{array}{l}\text { il a remplis } \\
\text { il a renvoyés }\end{array}$ & $\begin{array}{l}\text { de biens } \\
\text { vides }\end{array}$ & \\
\hline $\begin{array}{l}+{ }^{54} \mathrm{Il} \text { a secouru } \\
+ \text { pour se rappeler }\end{array}$ & $\begin{array}{l}\text { Israël } \\
\text { la miséricorde, }\end{array}$ & son serviteur, & \\
\hline $\begin{array}{l}:{ }^{55} \text { comme } \\
: \text { en-faveur-d'Abraham }\end{array}$ & $\begin{array}{l}\text { il avait dit } \\
\text { et de sa descendance }\end{array}$ & $\begin{array}{l}\text { à nos pères } \\
\text { à jamais. }\end{array}$ & \\
\hline
\end{tabular}

\section{c. Consigne}

\section{Premier regroupement de segments en morceaux :}

- Quels sont les segments qui forment une seule phrase?

- Quels sont les segments contigus qui sont parallèles entre eux?

- Réécrivez les morceaux que vous aurez identifiés, en commençant toujours par ce qui est le plus évident. D'un segment à l'autre à l'intérieur des morceaux, mettez en relief (caractères différents, couleurs...) non seulement les mots identiques mais aussi les synonymes. Attention, les rapports entre les membres du même segment ne doivent plus être visualisés, car cela a déjà été fait au niveau inférieur. N'oubliez pas les règles de réécriture des morceaux (Traité, 297-299; § 21 et 2.2). Écrivez aussi une brève description de la composition du morceau. 


\section{d. Solution}

- Dernier morceau (54-55) :

\begin{tabular}{lll}
\hline$+{ }^{54} \mathrm{Il}$ a secouru & ISRAËL & son serviteur, \\
- pour se rappeler & la miséricorde, & \\
$-{ }^{55}$ comme & il avait dit & à nos pères \\
+ en-faveur-d'ABRAHAM & et de sa descendance & à jamais.
\end{tabular}

Les deux segments forment une seule phrase complexe : principale (54a), finale (54b), comparative (55). Deux noms propres encadrent le morceau, «Israël » et « Abraham »; «Israël » est l'équivalent d' «Abraham et de sa descendance à jamais ». Les membres internes peuvent être dits en relation car ils se rapportent tous deux à la promesse passée.

- Premier morceau (46b-49a) :

\begin{tabular}{clll}
\hline$+{ }^{46 \mathrm{~b}}$ GRANDIT & mon âme & LE SEIGNEUR & \\
$+{ }^{47}$ et jubile & mon esprit & en Dieu & mon sauveur, \\
$:{ }^{48 \mathrm{a}}$ parce qu'il a regardé & 1'humiliation & de sa servante. & \\
$+{ }^{48 \mathrm{~b}}$ Voici en effet & à partir de maintenant & \\
+ me diront-heureuse & tous & les âges & \\
$:{ }^{49 \mathrm{a}}$ parce qu'il a fait à moi & de GRANDES (choses) & LE PUISSANT.
\end{tabular}

Ces deux segments trimembres sont parallèles: leur dernier membre qui commence avec "parce que » sont des causales dont le sujet est Dieu, «le Puissant». Ils sont complémentaires : dans le premier c'est Marie qui loue le Seigneur, dans le deuxième «tous les âges » à venir béniront Marie. "Grandit » et " grandes (choses») d'une part et « le Seigneur » et « le Puissant » de l'autre font inclusion.

. Un autre morceau (51-53) :

\begin{tabular}{|c|c|c|c|c|}
\hline$-{ }^{51}$ & $\begin{array}{l}\text { IL A FAIT } \\
\text { IL A DISPERSÉ }\end{array}$ & $\begin{array}{l}\text { force } \\
\text { LES OROUEILLEUX }\end{array}$ & $\begin{array}{l}\text { par son bras, } \\
\text { en pensée }\end{array}$ & de leur cœur; \\
\hline$-{ }^{52}$ & IL A RENVERSÉ & LES PUISSANTS & des trônes & \\
\hline+ et & IL A ÉLEVÉ & LES HUMILIES ; & & \\
\hline$+{ }^{53}$ & LES AFFAUIESS & IL A REMPLIS & de biens & \\
\hline - et & LES RICHES & IL A RENVOYÉS & vides. & \\
\hline
\end{tabular}

81 Chaque membre comporte un verbe aux mêmes modalités dont le sujet est le Seigneur. Dans les deux derniers segments qui sont coordonnés, les deux membres sont corrélés : c'est en renversant les puissants que Dieu élève les humiliés et, de manière spéculaire, c'est en vidant les riches qu'il comble les affamés. Le deuxième membre du premier segment montre comment Dieu traite «les orgueilleux», comme il fera pour «les puissants » et "les riches ». Quant au premier membre, même s'il ne nomme pas ceux contre qui Dieu « fait force », on comprend que c'est contre « les orgueilleux ».

\section{e. Consigne}

Regroupez les morceaux en parties :

- Que reste-t-il qui n'entre pas dans les trois morceaux analysés précédemment?

- Quelle est la spécificité de ce reste?

-Quels sont les points communs aux deux derniers morceaux?

\section{f. Solution}

Il ne reste qu'un seul segment trimembre : 


$\begin{array}{clll}-{ }^{49 b} & \text { Et saint } & \text { son nom } & \\ -50 & \text { et sa miséricorde } & \text { d'âges } & \text { en âges } \\ :: & \text { sur les craignant } & \text { lui. } & \end{array}$
morceaux sont au passé,

- quels sont les temps du premier morceau?

- quel est le temps du trimembre qui reste?

- enfin, quelle composition proposez-vous ? Faites la réécriture de l'ensemble en conséquence.

\section{h. Solution}

\begin{tabular}{|c|c|c|}
\hline \multicolumn{3}{|l|}{${ }^{46}$ Et Marie dit : } \\
\hline $\begin{array}{l}::{ }^{~} \text { Grandit } \\
::{ }^{47} \text { et jubile } \\
.48 \text { parce qu'il a regardé }\end{array}$ & $\begin{array}{l}\text { mon âme } \\
\text { mon esprit } \\
\text { L'HUMULIATION }\end{array}$ & $\begin{array}{l}\text { le Seigneur } \\
\text { en Dieu, mon sauveur, } \\
\text { de SA SERVANTE. }\end{array}$ \\
\hline $\begin{array}{l}:: \text { Voici en effet que } \\
:: \text { me diront-heureuse } \\
{ }_{49}^{49} \text { parce qu'll A FAIT à moi }\end{array}$ & $\begin{array}{l}\text { à partir de maintenan } \\
\text { tous les âges, } \\
\text { de grandes (choses) }\end{array}$ & LE PUISSANT. \\
\hline
\end{tabular}

\begin{tabular}{|c|c|c|}
\hline & $\begin{array}{l}\text { Et saint } \\
{ }_{50} \text { et SA MISÉRICORDE } \\
\text { sur les craignant }\end{array}$ & $\begin{array}{l}\text { son Nom } \\
\text { d'âge } \\
\text { lui. }\end{array}$ \\
\hline $\begin{array}{l}+{ }^{51} \text { IL A FAIT } \\
\text { - il a dispersé }\end{array}$ & $\begin{array}{l}\text { force } \\
\text { les orgueilleux }\end{array}$ & $\begin{array}{l}\text { par son bras, } \\
\text { en pensée de leur cœur; }\end{array}$ \\
\hline $\begin{array}{l}{ }^{52} \text { il a renversé } \\
+ \text { et il a élevé }\end{array}$ & $\begin{array}{l}\text { LES PUISSANTS } \\
\text { LES HUMIIES'S; }\end{array}$ & des trônes, \\
\hline $\begin{array}{l}+{ }^{53} \text { les affamés } \\
\text { - et les riches }\end{array}$ & $\begin{array}{l}\text { il a remplis } \\
\text { il a renvoyés }\end{array}$ & $\begin{array}{l}\text { de biens, } \\
\text { vides. }\end{array}$ \\
\hline $\begin{array}{l}::{ }^{54} \mathrm{Il} \text { a secouru } \\
:: \text { pour se rappeler }\end{array}$ & $\begin{array}{l}\text { Israël, } \\
\text { SA MISÉRICORDE, }\end{array}$ & SON SERVITEUR, \\
\hline $\begin{array}{l}={ }^{55} \text { comme } \\
=\text { à Abraham }\end{array}$ & $\begin{array}{l}\text { il l'avait dit } \\
\text { et à sa descendance }\end{array}$ & $\begin{array}{l}\text { à nos pères, } \\
\text { pour toujours. " }\end{array}$ \\
\hline
\end{tabular}


d'avoir été choisie pour être la mère du Sauveur, malgré la bassesse, l'humilité de sa condition. Il faut qu'elle le dise; et elle dit qu'elle le dit. Comme celui qui rit et qui est tellement heureux qu'il ne peut s'empêcher de dire qu'il rit ! Elle parle au présent, mais c'est pour rappeler un passé récent, celui de l'Annonciation et de la Visitation (Lc 1,26-45). Mais aussitôt, à partir du présent l'horizon s'élargit jusqu'à la fin des temps (48b-49a). Le second segment en effet part de "maintenant» comme le premier, et s'étend à l'infini, à "tous les âges». Une grande joie ne peut pas ne pas se communiquer et le lecteur - ou mieux, celui qui récite ou chante avec Marie son cantique - se trouve invité à entrer dans la danse : de «l'humiliation» le Seigneur a fait de "grandes choses ». Il les a faites pour Marie, dont l'âme ne peut que " grandir » le Seigneur, c'est-à-dire reconnaître et proclamer sa grandeur. Mais si nous entrons dans la danse, nous proclamons avec Marie qu'il les a faites pour «tous les âges », pour nous.

La troisième partie se tourne vers le passé, jusqu'à l'origine du peuple élu, jusqu'à Abraham. Les trois segments du premier morceau (51-53) développent la même idée fondamentale : le Seigneur a abaissé les puissants et au contraire élevé les petits. Ce sont trois variations sur la loi générale de la sagesse divine, synthétisée par les évangiles dans la formule plusieurs fois reprise: «Beaucoup de premiers seront derniers, et de derniers seront premiers " (Mt 19,30;20,16; Mc 10,31) ou "Qui s'élève sera abaissé et qui s'abaisse sera élevé » (Mt 23,12; Lc 14,11; 18,14). On remarquera cependant que le premier segment ne parle pas des humiliés, mais seulement des orgueilleux et qu'il commence par l'affirmation de la force du Seigneur : toute l'œuvre de salut trouvant sa source dans son bras, il fallait le proclamer d'entrée de jeu.

91 Le dernier morceau (54-55) applique la loi générale du morceau précédent au peuple d'Israël et à son histoire depuis son commencement en Abraham. Ainsi, alors que la première partie du chant regardait vers l'avenir «à partir de maintenant » jusqu'à la fin des temps, la dernière remonte dans le passé jusqu'à l'origine du peuple élu, «à Abraham ». Le dernier mot du cantique est «pour toujours». Le cantique de Marie va de toujours à toujours.

Mais ce n'est pas tout. De la première partie nous avons sauté à pieds joints vers la dernière, en passant par-dessus la partie centrale. Celle-ci est très courte, lapidaire : elle constitue le cœur du cantique, son noyau dur, sa clé de voûte, cette pierre savamment taillée qui assure la cohérence et la solidité de toute la construction. La première partie regardait vers le futur jusqu'à la fin, la dernière contemplait le passé jusqu'au commencement. Au centre, deux phrases nominales, des phrases sans un verbe qui préciserait le temps - ce sont les seules de tout le chant. Elles indiquent donc ce qui est de toujours; et du reste, elles le disent : «d'âge en âge » (ou « de génération en génération »). Comme dans le Sanctus ("Saint, saint, saint le Seigneur »; Is 6,3), le Nom de Dieu est d'abord proclamé, acclamé. Puis la sainteté est pour ainsi dire définie : elle se manifeste avant tout dans la "miséricorde». Ce mot reviendra à la fin, pour Israël depuis Abraham, mais la réalité était déjà présente dès le début : c'est dans sa miséricorde que le Seigneur « a regardé l'humiliation de sa servante ». Tout découle de l'amour - l'autre nom de la miséricorde -, l'amour de Dieu, qui est de toujours à 
toujours, mais encore faut-il qu'il soit accepté par les hommes, par «ceux qui le craignent ». La crainte de Dieu, ce n'est pas la peur, c'est « la certitude tremblante de l'amour ${ }^{17}$ ». À l'amour ne peut répondre que l'amour. Le Magnificat est un chant d'amour.

\section{Conclusion}

\section{NOTES}

1. R. Meynet - J. Oniszczuk, Exercices d'analyse rhétorique biblique, Pendé, Gabalda (Rhétorique Sémitique 12), 2013.

2. R. Meynet, "E ora, scrivete per voi questo cantico ». Introduzione pratica all'analisi retorica. I. Detti e proverbi, Rome, Edizioni Dehoniane (Retorica Biblica 3), 1996.

3. Voir R. Meynet, Traité de rhétorique biblique, deuxième édition revue et corrigée, Pendé, Gabalda (Rhétorique Sémitique 11), chap. 1, « Historique », «B. Les fondateurs », p. 49-83.

4. Th. Boys, A Key to the Book of the Psalms, Londres, L. B. Seeley \& Sons, 1825.

5. Ibid., «Exercices pour étudiants», p. 236-239, traduction R. Meynet. Les crochets droits signalent des ajouts du traducteur ; dans les références bibliques, les chiffres romains de Boys ont été transposés en chiffres arabes.

6. Référence actuelle : Mc 3, 20.

7. Voir R. Meynet, L'évangile de Marc, Pendé, Gabalda, 2014, p. 216.

8. Voir ibid., p. 412.

9. Voir ibid., séquence A3 (Mc 3,7-35), p. 97-118.

10. Voir ibid., p. 120.

11. Bible de Jérusalem (abrégée en BJ) 1956 : « sur son humble servante ».

12. BJ 1956 : « rassasié ».

13. BJ 1956 : «a porté secours ».

14. BJ 1956 : « ainsi qu'il l'avait promis ».

15. BJ 1956 : « descendance ».

16. Voir R. Meynet, L'évangile de Marc, op. cit., p. 327-370 ; dans ma traduction de chaque passage j'ai traduit tous les kai initiaux par « et », mais je n'ai pas traduit les de. 
17. P. Beauchamp, L'Un et l'Autre Testament. I. Essai de lecture, Paris, Le Seuil, 1976, p. 272 : «La certitude tremblante de l'amour, cela exactement que la Bible appelle "crainte de Dieu" ».

\section{AUTEUR}

\section{ROLAND MEYNET}

Professeur émérite de théologie biblique à la Faculté de théologie de l'Université Grégorienne de Rome, membre fondateur et Secrétaire de la RBS 